Journal of Patient-Centered

$1-25-2016$

\title{
Racial Disparities in Outcomes Following Kidney Transplantation: A Single-Center Experience
}

\author{
Vani Nilakantan \\ Maharaj Singh \\ Ruth M. Perez \\ Yang Shi \\ Ahmed Dalmar \\ Brittany T. Last \\ Ajay Sahajpal
}

Follow this and additional works at: https://aah.org/jpcrr

Part of the Clinical Epidemiology Commons, and the Endocrine System Diseases Commons

\section{Recommended Citation}

Nilakantan V, Singh M, Perez RM, Shi Y, Dalmar A, Last BT, Sahajpal A. Racial disparities in outcomes following kidney transplantation: a single-center experience. J Patient Cent Res Rev. 2016;3:9-19. doi: 10.17294/2330-0698.1203

Published quarterly by Midwest-based health system Advocate Aurora Health and indexed in PubMed Central, the Journal of Patient-Centered Research and Reviews (JPCRR) is an open access, peer-reviewed medical journal focused on disseminating scholarly works devoted to improving patient-centered care practices, health outcomes, and the patient experience. 


\title{
Racial Disparities in Outcomes Following Kidney Transplantation: A Single-Center Experience
}

\author{
Vani Nilakantan, PhD, ${ }^{1}$ Maharaj Singh, PhD, ${ }^{1}$ Ruth M. Perez, BA,${ }^{1}$ Yang Shi, PhD, ${ }^{1}$ \\ Ahmed Dalmar, MD, ${ }^{1}$ Brittany T. Last, BS, ${ }^{1}$ Ajay Sahajpal, MD ${ }^{2}$ \\ ${ }^{1}$ Aurora Research Institute, Aurora Health Care, Milwaukee, Wisconsin \\ ${ }^{2}$ Abdominal Transplant Surgery, Aurora Health Care, Milwaukee, Wisconsin
}

\begin{abstract}
Purpose Kidney transplantation remains the best treatment option for end-stage renal disease. However, despite overall improvements in patient and graft survival rates after kidney transplantation, differences in outcomes still exist among different racial and ethnic groups, with African-Americans having lower graft survival. Gaps continue to exist in the understanding of how demographic factors contribute to the varying outcomes among racial/ethnic groups.

Methods We retrospectively evaluated kidney transplant outcomes in four racial/ethnic groups over a 12-year period at a large tertiary care center. Primary and secondary study outcomes were patient and graft survival across groups. To determine factors that might predict posttransplant outcomes, we analyzed both demographic and clinical variables. Descriptive statistics, Kaplan-Meier survival function and Cox regression were used for analyses.

Results The two most common causes of end-stage renal disease across all groups were hypertension and diabetes, accounting for $45 \%$ of the transplant recipient population. Although patient survival was similar across the four racial/ethnic groups, at 5-year follow-up, graft survival was lower for African-Americans than Caucasians $(\mathrm{P}<0.05)$. Preprocedure heart failure, graft loss and hepatitis $\mathrm{C}$-positive donor status were associated with lower patient survival. Female gender, hepatitis C-positive donor, smoking history, preprocedure myocardial infarction, preprocedure cerebrovascular accident and elevated serum creatinine level contributed to significant graft loss.
\end{abstract}

Conclusions African-Americans have worse 5-year graft outcomes compared with Caucasians. This posttransplant outcome was multifactorial and no individual factor could be singled out to explain poorer outcomes in African-Americans. Prospective studies are needed to further delineate the causes of graft loss, and interventional studies will be needed to improve outcomes for African-American kidney transplant recipients. (J Patient-Centered Res Rev. 2016;3:9-19.)

Keywords race; disparities; kidney transplantation; survival; hypertension; diabetes

Kidney transplantation has long been considered the optimal treatment for end-stage renal disease. While artificial replacement of lost kidney function by dialysis gives patients with end-stage renal disease an additional 8-year life expectancy on average, this number can grow to 20 or more with a transplant. ${ }^{1,2}$ Incidence rates are more than three times higher for African-Americans than for Caucasians and have risen more quickly than all other races. ${ }^{3}$ For more than two

Correspondence: Vani Nilakantan, PhD,

Aurora Research Institute, 960 N. 12th Street, \#4120,

Milwaukee, WI, 53233, T: 414-219-7846, F: 414-219-5381,

Email: vani.nilakantan@aurora.org decades, the transplant community has been aware of racial disparities in patient outcomes after kidney transplantation. ${ }^{4}$ Although overall outcomes have steadily improved over the last decade, gaps across different racial/ethnic groups still remain.

Barriers exist at different levels of the transplant process and may be responsible for the poorer outcomes seen in African-Americans after transplantation. Access to transplant centers might be a contributing factor to posttransplant outcomes, specifically in relation to ease of patient access (center characteristics, proximity to patients, etc.) and the variability in shipping donated organs. ${ }^{5}$ It is often more difficult for African-Americans than Caucasians to access a transplant, even when the 
former are on the kidney transplant waiting list, due to match criteria, severity of disease and regional policies. ${ }^{6,7}$ In 2001, nearly $70 \%$ of Caucasian patients on the list received a transplant within 5 years, whereas only $54 \%$ of African-Americans received a transplant over the same time period. ${ }^{5}$ Even after receiving renal transplants, African-American patients fare substantially worse long term than Caucasians or other minorities. Survival rates in African-Americans drop more progressively ( $90 \%$ at 1 year, $73 \%$ at 3 years and $59 \%$ at 5 years) compared with Caucasians ( $92 \%, 84 \%$ and $76 \%$, respectively). ${ }^{8,9}$ Similarly, while the overall rate of graft survival from both deceased and living donor renal transplants has improved in the past decade by approximately $13 \%$ and $7 \%$, respectively, AfricanAmericans continue to have worse graft function and shorter graft survival than Caucasians. ${ }^{10-12}$

Studies have suggested multiple factors - comorbid conditions, higher immunological risks and other biological and socioeconomic factors - may contribute to disparities in kidney transplant outcomes. ${ }^{13,14}$ For example, worse outcomes for African-American transplant recipients have been attributed to poor education, socioeconomic status, cultural influences, lack of continuous care, etc. ${ }^{15-17}$ In a study of transplant recipients within the Veterans Affairs health system, where access to care is equivalent for all patients, large racial disparities in kidney transplant outcomes were still observed. ${ }^{18}$ Even when access to medications was essentially guaranteed, poorer outcomes in AfricanAmericans versus Caucasians were documented, with more rejection and allograft failure despite comparable immunosuppression. ${ }^{19}$ In 2006, Goldfarb-Rumyantev et al. showed significantly better outcomes for patients who had private insurance than those with Medicare. ${ }^{20}$ Other studies have shown that an adjustment for a number of demographic factors (e.g. socioeconomic or insurance status) could not fully explain the differences observed between racial/ethnic groups ${ }^{21}$ and that race/ ethnicity remains a significant factor affecting graft survival. ${ }^{22}$

As both physiological and environmental factors contribute significantly to disparities in outcomes, understanding and dissecting the determinants that unfairly distribute the burden of disease in kidney transplant recipients is critical to ensure equitable transplant access and outcomes. The purpose of this study is to identify the risk factors that can adversely affect kidney transplant outcomes in a single transplant center, and specifically highlight areas suited for targeted interventions that would improve outcomes across four racial/ethnic groups defined as AfricanAmerican, Caucasian, Hispanic and Other.

\section{METHODS}

Retrospective chart review of all adult kidney transplant recipients at a single hospital in our health system from January 1, 1999, to December 31, 2013, was approved by the Institutional Review Board. Initially, 381 primary kidney transplants were identified through the Organ Procurement and Transplantation Network. Patients with a history of multiple organ transplantation, prior renal transplantation or those who died within 30 days after transplantation were excluded, as were duplicated patient entries. One patient with 23 years of dialysis was excluded as an outlier. A total of 307 unique patients comprised the study sample (Figure 1).

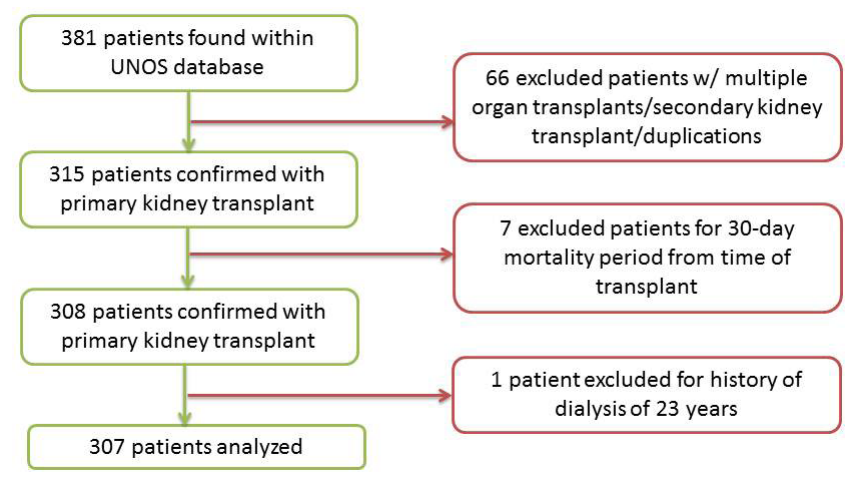

Figure 1. Cohort identification of study sample. UNOS, United Network for Organ Sharing.

Self-reported race/ethnicity registries were subdivided into Caucasian, African-American, Hispanic and Other (Asians, Pacific Islanders, Native Americans, Alaskan Natives, etc.). Body mass index (BMI) was calculated using height and weight at the time of procedure, and also at $0-3$ years, 3-5 years and 5-10 years after procedure (if information was available). Date of birth was collected and used to calculate recipient age at time of transplant. Comorbidities, including history of hypertension, diabetes, hyperlipidemia, myocardial infarction, heart failure, cerebrovascular accident and coronary artery disease prior to transplantation, were 
based on ICD-9 codes. Similarly, follow-up data regarding these comorbidities were collected for all patients at $0-1$ years and $1-3$ years posttransplant.

Clinical data for time on dialysis, length of hospital stay, blood urea nitrogen (BUN) level, glomerular filtration rate and creatinine level were recorded at baseline, time of discharge and 1 week, 30 days, 3 months, 1 year and 3 years posttransplant.

The primary outcome for this study was patient survival as determined by all-cause mortality. The secondary outcome was graft survival, and the return of a patient to hemodialysis or peritoneal dialysis on a permanent basis was recorded as graft loss. The association of race with patient and graft survival in the overall cohort was examined among the four groups.

\section{Statistical Analysis}

All categorical variables were summarized using frequency and percentage. Percentages were compared across the four racial/ethnic groups using chi-square or Fisher's exact test. Data are summarized as mean \pm standard deviation for continuous variables, and Analysis of Variance (ANOVA) was used to compare means across the four groups. Additional multiple comparison tests were used to compare paired groups. Cumulative probabilities for patient and graft survival were estimated using Kaplan-Meier survival function. Patient and graft survival rates were compared across the four groups using log-rank test. Mean patient and graft survival years also were calculated for all races. The Cox proportional hazards model was used to determine the risk factors for patients as well as for graft survival. For all statistical tests, significance was set at $\mathrm{P}<0.05$. All statistical analyses were performed using SAS 9.4 (SAS Institute Inc., Cary, NC).

\section{RESULTS \\ Patient Characteristics}

The most prominent causes of end-stage renal disease, as collected from United Network for Organ Sharing, were diabetic nephropathy (type I and type II), hypertensive nephrosclerosis and polycystic kidney disease (Figure 2). Diabetic nephropathy and hypertensive nephrosclerosis contributed to almost half the end-stage renal disease population (45\%).

Demographics and baseline characteristics of the patient population are summarized by race in Table 1 . The study cohort was evenly distributed between age groups, was $58 \%$ male and was $61 \%$ Caucasian. The majority of donors were Caucasian (76\%) as well as cytomegalovirus (CMV)-negative (52\%) and hepatitis C (HepC)-negative (98\%). Most patients suffered from hypertension (93\%), hyperlipidemia (72\%) and diabetes (56\%). Patient proportions were different across the four races for the following variables: donor race, donor CMV status, recipient CMV status, recipient HepC status, delayed graft function, BMI, alcohol and other drug abuse, pretransplant diabetes and 1-year posttransplant diabetes. Specifically, there was a higher proportion of Caucasian donors than any other group, alcohol/drug abuse history was higher in Caucasians (42\%), African-Americans (49\%) and Hispanics (25\%) compared with Other (12\%), and

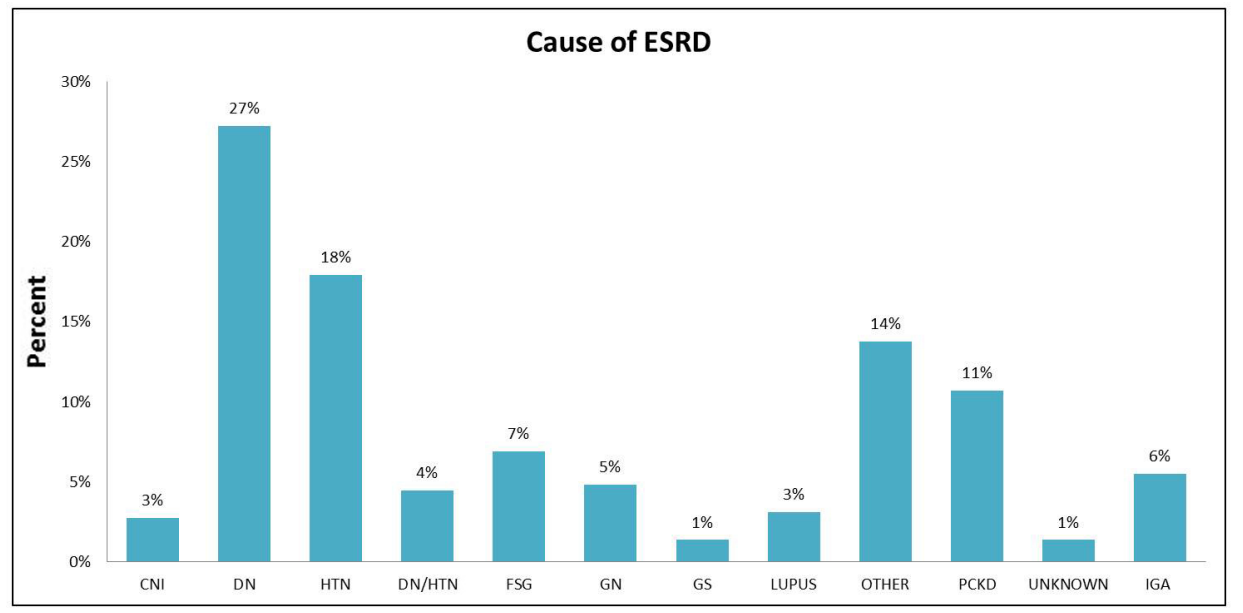

Figure 2. Causes of endstage renal disease (ESRD) for study cohort. CNI, calcineurin inhibitor toxicity; $D N$, diabetic nephropathy; HTN, hypertensive nephrosclerosis; FSG, focal glomerulosclerosis; GN, glomerulonephritis; GS, glomerulosclerosis; PCKD, polycystic kidney disease; IGA, immunoglobulin A nephropathy. 
Table 1. Distribution of population characteristics across all races by frequency and percentage

\begin{tabular}{|c|c|c|c|c|c|c|}
\hline \multirow[b]{2}{*}{ Patient characteristics } & \multirow[b]{2}{*}{$\begin{array}{c}\text { Total } \\
\mathrm{N}=307\end{array}$} & \multicolumn{4}{|c|}{ Race/Ethnicity } & \multirow[b]{2}{*}{$P^{*}$} \\
\hline & & $\begin{array}{c}\text { CAU } \\
186(61 \%)\end{array}$ & $\begin{array}{c}\text { AA } \\
59(19 \%)\end{array}$ & $\begin{array}{c}\text { HIS } \\
44(14 \%)\end{array}$ & $\begin{array}{c}\text { Other } \\
18(6 \%)\end{array}$ & \\
\hline Age & & & & & & 0.207 \\
\hline 19-39 years & $48(16 \%)$ & $23(12 \%)$ & $9(15 \%)$ & $10(23 \%)$ & $6(33 \%)$ & \\
\hline 40-49 years & $61(20 \%)$ & $33(18 \%)$ & $16(27 \%)$ & $8(18 \%)$ & $4(22 \%)$ & \\
\hline $50-59$ years & $81(26 \%)$ & $59(32 \%)$ & $10(17 \%)$ & $10(23 \%)$ & $2(11 \%)$ & \\
\hline $60-69$ years & $80(26 \%)$ & $48(26 \%)$ & $17(29 \%)$ & $12(27 \%)$ & $3(17 \%)$ & \\
\hline$\geq 70$ years & $37(12 \%)$ & $23(12 \%)$ & $7(12 \%)$ & $4(9 \%)$ & $3(17 \%)$ & \\
\hline Gender & & & & & & 0.1098 \\
\hline Female & $130(42 \%)$ & $72(39 \%)$ & $28(47 \%)$ & $18(41 \%)$ & $12(67 \%)$ & \\
\hline Male & $177(58 \%)$ & $114(61 \%)$ & $31(53 \%)$ & $26(59 \%)$ & $6(33 \%)$ & \\
\hline Donor race & & & & & & $<0.0001^{1}$ \\
\hline CAU & $233(76 \%)$ & $161(87 \%)$ & $36(61 \%)$ & $22(50 \%)$ & $14(78 \%)$ & \\
\hline AA & $38(12 \%)$ & $14(8 \%)$ & $17(29 \%)$ & $5(11 \%)$ & $2(11 \%)$ & \\
\hline HIS & $30(10 \%)$ & $8(4 \%)$ & $6(10 \%)$ & $16(36 \%)$ & 0 & \\
\hline OTH & $6(2 \%)$ & $3(2 \%)$ & 0 & $1(2 \%)$ & $2(11 \%)$ & \\
\hline Donor CMV & & & & & & $<0.0001^{2}$ \\
\hline Positive & $130(48 \%)$ & $74(47 \%)$ & $31(55 \%)$ & $25(64 \%)$ & 0 & \\
\hline Negative & $141(52 \%)$ & $84(53 \%)$ & $25(45 \%)$ & $14(36 \%)$ & $18(100 \%)$ & \\
\hline Recipient CMV & & & & & & $<0.0001^{3}$ \\
\hline Positive & $170(62 \%)$ & $81(49 \%)$ & $36(75 \%)$ & $38(88 \%)$ & $15(94 \%)$ & \\
\hline Negative & $104(38 \%)$ & $86(51 \%)$ & $12(12 \%)$ & $5(12 \%)$ & $1(6 \%)$ & \\
\hline Donor hepatitis C & & & & & & 0.079 \\
\hline Positive & $6(2 \%)$ & $1(1 \%)$ & $3(5 \%)$ & $2(5 \%)$ & 0 & \\
\hline Negative & $299(98 \%)$ & $183(99 \%)$ & $56(95 \%)$ & $42(95 \%)$ & $18(100 \%)$ & \\
\hline Recipient hepatitis C & & & & & & $0.0131^{4}$ \\
\hline Positive & $10(4 \%)$ & $2(1 \%)$ & $5(10 \%)$ & $3(7 \%)$ & 0 & \\
\hline Negative & $262(96 \%)$ & $163(99 \%)$ & $44(90 \%)$ & $39(93 \%)$ & $16(100 \%)$ & \\
\hline Delayed graft function & & & & & & $0.011^{4}$ \\
\hline Yes & $51(18 \%)$ & $22(13 \%)$ & $17(33 \%)$ & $9(20 \%)$ & $3(18 \%)$ & \\
\hline No & $231(82 \%)$ & $148(87 \%)$ & $34(67 \%)$ & $35(80 \%)$ & $14(82 \%)$ & \\
\hline BMI (at procedure) & & & & & & $0.014^{5}$ \\
\hline Underweight/normal $(<25)$ & $72(24 \%)$ & $38(21 \%)$ & $16(27 \%)$ & $9(21 \%)$ & $9(53 \%)$ & \\
\hline Overweight (25-29.9) & $97(33 \%)$ & $52(29 \%)$ & $21(36 \%)$ & $18(43 \%)$ & $6(35 \%)$ & \\
\hline Obese $(\geq 30)$ & $129(43 \%)$ & $90(50 \%)$ & $22(37 \%)$ & $15(36 \%)$ & $2(12 \%)$ & \\
\hline Employment & & & & & & 0.325 \\
\hline Yes & $131(45 \%)$ & $84(48 \%)$ & $25(86 \%)$ & $14(33 \%)$ & $8(44 \%)$ & \\
\hline Education & & & & & & $<0.0001^{6}$ \\
\hline Less than high school & $20(7 \%)$ & $4(2 \%)$ & $2(4 \%)$ & $12(31 \%)$ & $2(14 \%)$ & \\
\hline High school and above & $250(93 \%)$ & $161(98 \%)$ & $50(96 \%)$ & 27 (69\%) & $12(86 \%)$ & \\
\hline Smoking & & & & & & 0.306 \\
\hline Yes & $145(48 \%)$ & $89(49 \%)$ & $32(55 \%)$ & $16(36 \%)$ & $8(47 \%)$ & \\
\hline AODA & & & & & & $0.007^{7}$ \\
\hline Yes & $118(39 \%)$ & $77(42 \%)$ & $28(49 \%)$ & $11(25 \%)$ & $2(12 \%)$ & \\
\hline Transplant type & & & & & & 0.069 \\
\hline Cadaveric & $199(69 \%)$ & $111(62 \%)$ & $42(82 \%)$ & $32(73 \%)$ & $14(82 \%)$ & \\
\hline Living related donor & $56(19 \%)$ & $38(21 \%)$ & $7(14 \%)$ & $9(20 \%)$ & $2(12 \%)$ & \\
\hline Living unrelated donor & $35(12 \%)$ & $29(16 \%)$ & $2(4 \%)$ & $3(7 \%)$ & $1(6 \%)$ & \\
\hline
\end{tabular}


Table 1 (cont). Distribution of population characteristics across all races by frequency and percentage

\begin{tabular}{|c|c|c|c|c|c|c|}
\hline \multirow[b]{2}{*}{ Patient characteristics } & \multirow[b]{2}{*}{$\begin{array}{c}\text { Total } \\
\mathrm{N}=307\end{array}$} & \multicolumn{4}{|c|}{ Race/Ethnicity } & \multirow[b]{2}{*}{$P^{*}$} \\
\hline & & $\begin{array}{c}\text { CAU } \\
186(61 \%)\end{array}$ & $\begin{array}{c}\text { AA } \\
59(19 \%)\end{array}$ & $\begin{array}{c}\text { HIS } \\
44(14 \%)\end{array}$ & $\begin{array}{c}\text { Other } \\
18(6 \%)\end{array}$ & \\
\hline Graft loss & & & & & & 0.1203 \\
\hline Yes & $31(11 \%)$ & $15(9 \%)$ & $9(19 \%)$ & $3(8 \%)$ & $4(24 \%)$ & \\
\hline Patient status & & & & & & 0.990 \\
\hline Alive & $262(85 \%)$ & $159(85 \%)$ & $50(83 \%)$ & $38(86 \%)$ & $15(83 \%)$ & \\
\hline Expired & $45(15 \%)$ & $27(15 \%)$ & $9(18 \%)$ & $6(14 \%)$ & $3(17 \%)$ & \\
\hline 30-day readmission & & & & & & 0.966 \\
\hline Yes & $85(31 \%)$ & $53(31 \%)$ & $16(33 \%)$ & $11(28 \%)$ & $5(31 \%)$ & \\
\hline Medication adherence & & & & & & 0.815 \\
\hline Yes & $117(38 \%)$ & $68(37 \%)$ & $22(37 \%)$ & $19(43 \%)$ & $8(44 \%)$ & \\
\hline \multicolumn{7}{|l|}{ Hypertension } \\
\hline Pre: Yes & $281(93 \%)$ & $169(92 \%)$ & $51(93 \%)$ & $44(100 \%)$ & $17(94 \%$ & 0.275 \\
\hline Post (0-1 years): Yes & $246(84 \%)$ & $147(82 \%)$ & $46(85 \%)$ & $37(86 \%)$ & $16(89 \%)$ & 0.601 \\
\hline Post (1-3 years): Yes & $236(83 \%)$ & $144(82 \%)$ & $46(92 \%)$ & $33(79 \%)$ & $13(76 \%)$ & 0.251 \\
\hline \multicolumn{7}{|l|}{ Diabetes } \\
\hline Pre: Yes & $169(56 \%)$ & $95(52 \%)$ & $33(60 \%)$ & $35(80 \%)$ & $6(33 \%)$ & $0.002^{8}$ \\
\hline Post (0-1 years): Yes & $133(45 \%)$ & $77(43 \%)$ & $24(44 \%)$ & $27(63 \%)$ & $5(28 \%)$ & $0.049^{9}$ \\
\hline Post (1-3 years): Yes & $138(49 \%)$ & $79(45 \%)$ & $26(52 \%)$ & $27(64 \%)$ & $6(35 \%)$ & 0.099 \\
\hline \multicolumn{7}{|l|}{ Hyperlipidemia } \\
\hline Pre: Yes & $217(72 \%)$ & $137(75 \%)$ & $35(64 \%)$ & $35(80 \%)$ & $10(56 \%)$ & 0.096 \\
\hline Post (0-1 years): Yes & $147(50 \%)$ & 87 (49\%) & $28(52 \%)$ & $25(58 \%)$ & $7(39 \%)$ & 0.524 \\
\hline Post (1-3 years): Yes & $161(57 \%)$ & $99(57 \%)$ & $29(58 \%)$ & $23(55 \%)$ & $10(59 \%)$ & 0.988 \\
\hline \multicolumn{7}{|l|}{ Myocardial infarction } \\
\hline Pre: Yes & $27(9 \%)$ & $16(9 \%)$ & $3(6 \%)$ & $5(11 \%)$ & $3(18 \%)$ & 0.455 \\
\hline Post (0-1 years): Yes & $4(1 \%)$ & $1(1 \%)$ & $2(4 \%)$ & $1(2 \%)$ & 0 & 0.290 \\
\hline Post (1-3 years): Yes & $1(0.4 \%)$ & $1(1 \%)$ & 0 & 0 & 0 & 0.895 \\
\hline \multicolumn{7}{|l|}{ Heart failure } \\
\hline Pre Yes & $95(32 \%)$ & $55(30 \%)$ & $21(39 \%)$ & $14(32 \%)$ & $5(29 \%)$ & 0.683 \\
\hline Post (0-1 years): Yes & $37(13 \%)$ & $20(11 \%)$ & $11(21 \%)$ & $5(12 \%)$ & $1(6 \%)$ & 0.214 \\
\hline Post (1-3 years): Yes & $38(14 \%)$ & $19(11 \%)$ & $10(21 \%)$ & $7(17 \%)$ & $2(13 \%)$ & 0.305 \\
\hline \multicolumn{7}{|l|}{ Cerebrovascular accident } \\
\hline Pre: Yes & $58(20 \%)$ & $34(19 \%)$ & $12(22 \%)$ & $11(25 \%)$ & $1(6 \%)$ & 0.368 \\
\hline Post (0-1 years): Yes & $12(4 \%)$ & $7(4 \%)$ & $2(4 \%)$ & $3(7 \%)$ & 0 & 0.649 \\
\hline Post (1-3 years): Yes & $15(5 \%)$ & $7(4 \%)$ & $5(10 \%)$ & $3(7 \%)$ & 0 & 0.236 \\
\hline \multicolumn{7}{|l|}{ Coronary artery disease } \\
\hline Pre Yes & $140(47 \%)$ & $86(47 \%)$ & $22(41 \%)$ & $25(57 \%)$ & $7(41 \%)$ & 0.426 \\
\hline Post (0-1 years): Yes & $90(31 \%)$ & $52(29 \%)$ & $15(29 \%)$ & $16(37 \%)$ & $7(41 \%)$ & 0.566 \\
\hline Post (1-3 years): Yes & $82(29 \%)$ & $50(29 \%)$ & $14(29 \%)$ & $14(33 \%)$ & $4(24 \%)$ & 0.886 \\
\hline
\end{tabular}

${ }^{*} P$-value illustrates comparison of the proportion across all races. For variables with $P<0.05$, corresponding footnote details between which races significance was found.

Comparisons with statistical significance of $P<0.05:{ }^{1} C A U$ vs. $A A, C A U$ vs. HIS, AA vs. HIS, AA vs. Other, HIS vs. Other; ${ }^{2} \mathrm{CAU}$ vs. Other, AA vs. Other, HIS vs. Other; ${ }^{3} \mathrm{CAU}$ vs. AA, CAU vs. HIS, CAU vs. Other; ${ }^{4} \mathrm{CAU}$ vs. AA, CAU vs. Other; ${ }^{5} \mathrm{CAU}$ vs. AA; ${ }^{6} \mathrm{CAU}$ vs. HIS; ${ }^{7} \mathrm{CAU}$ vs. HIS, CAU vs. Other, AA vs. HIS, AA vs. Other; ${ }^{8} \mathrm{CAU}$ vs. HIS, AA vs. HIS, AA vs. Other, HIS vs. Other; ${ }^{9} \mathrm{HIS}$ vs. Other.

AA, African-American; AODA, alcohol and other drug abuse; BMI, body mass index; CAU, Caucasian;

CMV, cytomegalovirus; HIS, Hispanic. 
both African-Americans and Caucasians had more high school education and above compared with Hispanics. Very high proportions of Caucasians (79\%), African-Americans (73\%) and Hispanics (79\%) were either overweight (BMI 25-29.9) or obese (BMI $\geq 30$ ) compared with Other (47\%). Pretransplant diabetes was significantly higher in Hispanics compared with
African-Americans and Caucasians, and Other had a lower proportion of diabetics compared with the rest of the groups. In the time period immediately following transplantation ( $0-1$ years), the proportion of Hispanics with diabetes was higher than African-Americans, Caucasians or Other.

Table 2. Descriptive statistics for all races using ANOVA*

\begin{tabular}{|c|c|c|c|c|c|}
\hline $\begin{array}{l}\text { Patient } \\
\text { characteristics }\end{array}$ & $\begin{array}{c}\text { Caucasian } \\
\mathbf{N}(\text { mean } \pm \text { SD) }\end{array}$ & $\begin{array}{c}\text { African-American } \\
\text { N (mean } \pm \text { SD) }\end{array}$ & $\begin{array}{c}\text { Hispanic } \\
\text { N (mean } \pm \text { SD) }\end{array}$ & $\begin{array}{c}\text { Other } \\
\mathrm{N}(\text { mean } \pm \mathrm{SD})\end{array}$ & $\begin{array}{c}P \\
\text { (all races) }\end{array}$ \\
\hline Age, years & $186(56.0 \pm 12.6)$ & $59(53.9 \pm 13.7)$ & $44(52.6 \pm 15.2)$ & $18(48.3 \pm 18.0)$ & 0.0788 \\
\hline Procedure BMI & $180(30.4 \pm 6.6)$ & $59(28.8 \pm 5.8)$ & $42(28.8 \pm 4.1)$ & $17(25.2 \pm 4.1)$ & $0.004^{1}$ \\
\hline BMI: 0-3 years & $180(31.7 \pm 7.4)$ & $58(30.6 \pm 6.4)$ & $43(29.8 \pm 5.6)$ & $18(26.3 \pm 4.3)$ & $0.0101^{1}$ \\
\hline BMI: 3-5 years ${ }^{\dagger}$ & $109(32.0 \pm 7.4)$ & $33(31.0 \pm 5.8)$ & $28(31.3 \pm 5.7)$ & $8(28.2 \pm 3.5)$ & 0.4300 \\
\hline BMI: 5-10 years ${ }^{\dagger}$ & $87(31.6 \pm 7.2)$ & $21(30.2 \pm 5.7)$ & $20(30.6 \pm 5.7)$ & $6(26.4 \pm 5.2)$ & 0.2899 \\
\hline Time on dialysis, days & $137(770.1 \pm 571.5)$ & $47(1097.4 \pm 693.2)$ & $42(897.8 \pm 559.6)$ & $16(1340.6 \pm 721.6)$ & $0.0003^{1,2}$ \\
\hline Length of stay, days & $186(8.7 \pm 8.1)$ & $59(9.6 \pm 6.2)$ & $44(7.9 \pm 5.0)$ & $18(7.3 \pm 3.8)$ & 0.5641 \\
\hline Pre-BUN, md/dL & $165(49 \pm 22.0)$ & $52(42.3 \pm 19.5)$ & $38(47.2 \pm 18.6)$ & $17(48.1 \pm 21.9)$ & 0.2717 \\
\hline Discharge BUN & $164(47.0 \pm 22.7)$ & $57(48.6 \pm 28.2)$ & $38(43.7 \pm 22.5)$ & $18(41.1 \pm 25.7)$ & 0.5854 \\
\hline 1-week BUN & $165(40.5 \pm 24.5)$ & $52(51.5 \pm 30.0)$ & $38(36.6 \pm 24.1)$ & $16(38.3 \pm 21.9)$ & $0.0217^{1,3}$ \\
\hline 30-day BUN & $169(26.6 \pm 13.4)$ & $57(23.6 \pm 8.1)$ & $39(23.4 \pm 7.4)$ & $17(27.3 \pm 11.7)$ & 0.2089 \\
\hline 3-month BUN & $167(25.6 \pm 12.4)$ & $56(24.6 \pm 9.6)$ & $41(23.6 \pm 8.3)$ & $16(22.1 \pm 5.8)$ & 0.5037 \\
\hline 1-year BUN & $172(24.5 \pm 10.8)$ & $56(24.4 \pm 11.8)$ & $44(23.4 \pm 9.4)$ & $16(17.9 \pm 5.8)$ & 0.1257 \\
\hline 3-year BUN ${ }^{\dagger}$ & $140(24.6 \pm 11.8)$ & $39(23.8 \pm 11.6)$ & $39(23.7 \pm 10.0)$ & $15(19.3 \pm 7.9)$ & 0.3938 \\
\hline Pre-creatinine, mg/dL & $183(6.7 \pm 2.8)$ & $53(9.6 \pm 4.0)$ & $44(8.1 \pm 3.4)$ & $17(9.2 \pm 7.9)$ & $0.0001^{1,2}$ \\
\hline Discharge creatinine & $173(3.6 \pm 2.6)$ & $57(6.1 \pm 4.3)$ & $39(3.9 \pm 3.3)$ & $18(3.5 \pm 2.5)$ & $0.0001^{1,3,4}$ \\
\hline 1-week creatinine & $168(2.5 \pm 2.0)$ & $52(4.4 \pm 3.6)$ & $38(2.5 \pm 2.9)$ & $16(2.5 \pm 1.6)$ & $0.0001^{1,3,4}$ \\
\hline 30-day creatinine & $171(1.6 \pm 1.2)$ & $57(1.8 \pm 1.4)$ & $40(1.4 \pm 1.3)$ & $17(1.5 \pm 0.8)$ & 0.4216 \\
\hline 3-month creatinine & $168(1.5 \pm 0.8)$ & $56(1.6 \pm 0.8)$ & $41(1.4 \pm 1.3)$ & $16(1.2 \pm 0.3)$ & 0.278 \\
\hline 1-year creatinine & $172(1.5 \pm 0.8)$ & $56(1.9 \pm 1.7)$ & $44(1.5 \pm 1.4)$ & $16(1.2 \pm 0.3)$ & $0.0489^{4}$ \\
\hline 3 -year creatinine ${ }^{\dagger}$ & $139(1.6 \pm 1.4)$ & $40(2.0 \pm 1.8)$ & $39(1.4 \pm 0.6)$ & $15(1.2 \pm 0.5)$ & 0.1497 \\
\hline Pre-GFR ${ }^{\dagger}$ & $117(11.0 \pm 7.2)$ & $33(8.2 \pm 4.6)$ & $26(8.3 \pm 4.8)$ & $14(10.1 \pm 13.9)$ & 0.1491 \\
\hline Discharge GFR ${ }^{\dagger}$ & $119(28.2 \pm 19.3)$ & $31(20.5 \pm 20.0)$ & $26(24.9 \pm 17.2)$ & $15(28.1 \pm 20.4)$ & 0.2445 \\
\hline 1-week GFR ${ }^{\dagger}$ & $119(39.3 \pm 17.5)$ & $36(28.3 \pm 20.3)$ & $26(39.1 \pm 18.7)$ & $14(35.0 \pm 17.5)$ & $0.0163^{2}$ \\
\hline 30-day GFR ${ }^{\dagger}$ & $120(50.0 \pm 12.0)$ & $41(47.5 \pm 13.7)$ & $27(51.8 \pm 11.1)$ & $15(48.7 \pm 14.7)$ & 0.5432 \\
\hline 3-month GFR ${ }^{\dagger}$ & $117(51.1 \pm 10.7)$ & $39(50.3 \pm 11.8)$ & $28(53.8 \pm 10.6)$ & $14(55.1 \pm 5.9)$ & 0.3051 \\
\hline 1-year GFR ${ }^{\dagger}$ & $124(51.2 \pm 10.6)$ & $44(49.0 \pm 13.6)$ & $32(51.1 \pm 11.0)$ & $13(52.2 \pm 7.8)$ & 0.6743 \\
\hline 3-year GFR ${ }^{\dagger}$ & $109(49.8 \pm 12.2)$ & $37(48.1 \pm 14.9)$ & $33(51.6 \pm 11.0)$ & $14(51.6 \pm 11.2)$ & 0.6545 \\
\hline 1-month tacrolimus & $162(9.5 \pm 3.3)$ & $55(8.9 \pm 3.9)$ & $38(9.9 \pm 3.2)$ & $16(10.6 \pm 3.5)$ & 0.2952 \\
\hline 3-month tacrolimus & $159(9.0 \pm 3.1)$ & $54(8.4 \pm 2.5)$ & $40(9.2 \pm 3.1)$ & $16(8.4 \pm 2.7)$ & 0.517 \\
\hline 1-year tacrolimus & $168(7.9 \pm 2.5)$ & $52(8.1 \pm 2.9)$ & $43(8.5 \pm 4.0)$ & $16(7.3 \pm 2.4)$ & 0.4531 \\
\hline 3-year tacrolimus ${ }^{\dagger}$ & $137(6.8 \pm 2.2)$ & $38(6.1 \pm 1.8)$ & $38(6.4 \pm 2.3)$ & $15(5.8 \pm 2.7)$ & 0.1773 \\
\hline
\end{tabular}

${ }^{*}$ Post-hoc analysis used on variable with $P<0.05$ to determine which paired races were statistically different. †Variables contained more than $20 \%$ missing data.

${ }^{1}$ Caucasian vs. Other; ${ }^{2}$ Caucasian vs. African-American; ${ }^{3}$ African-American vs. Hispanic; ${ }^{4}$ African-American vs. Other. BMI, body mass index; BUN, blood urea nitrogen; GFR, glomerular filtration rate; $S D$, standard deviation. 
Figure 3. Survival probability (A) for patients having undergone kidney transplant with number of subjects at risk across all races $(P=0.824)$. Graft survival ( $B$ ) depicted by log-rank tests for the same patient cohort along with subjects at risk across all races $(P<0.04)$ with significance between Caucasians and African-Americans and between Caucasians and Other $(P<0.05)$. AA, AfricanAmerican; CAU, Caucasian; HIS, Hispanic; OTH, Other.
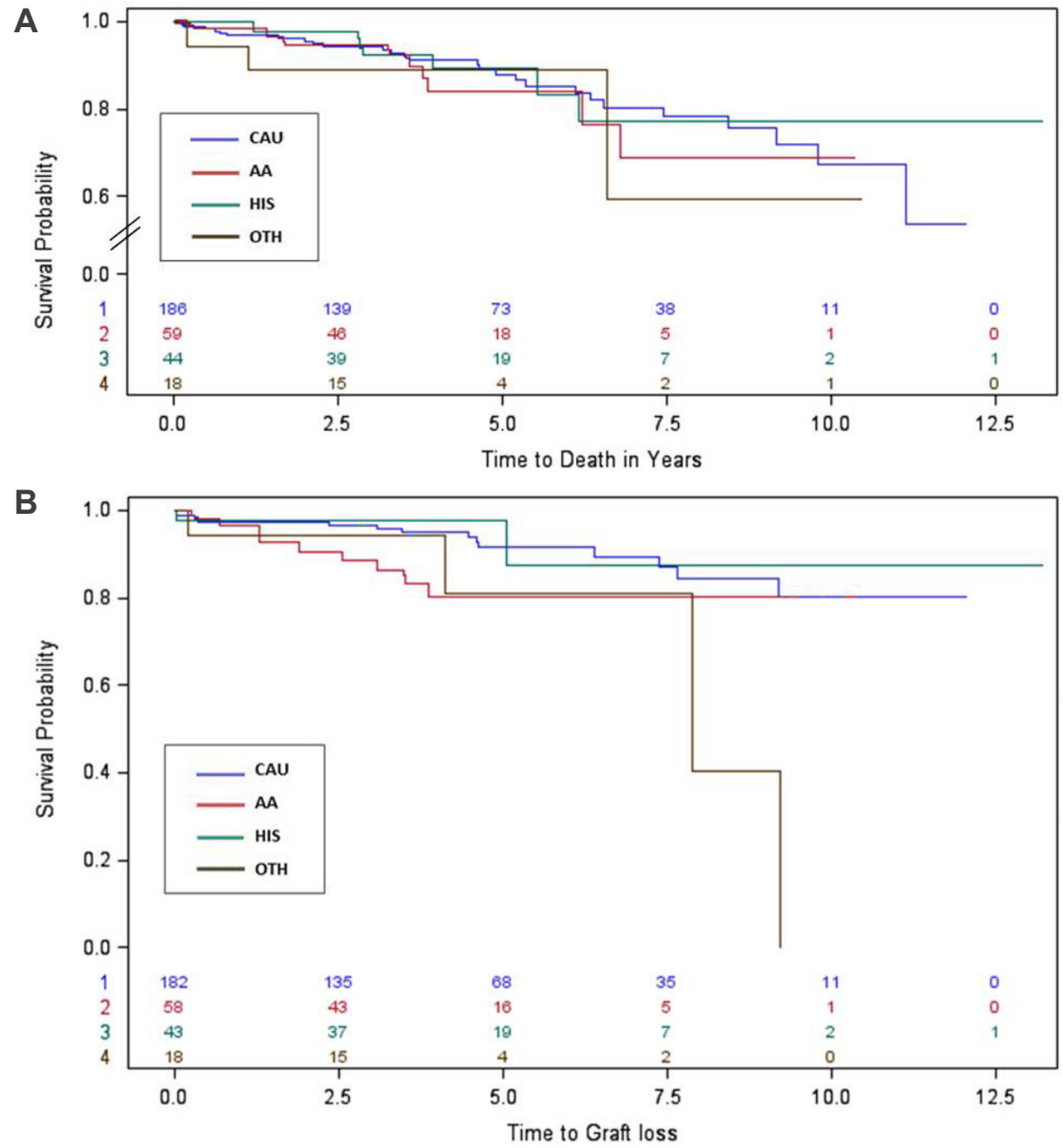

Clinical characteristics of the patient population are shown in Table 2. Mean time on dialysis was significantly higher for African-Americans (1097.4 \pm 693.2 days) and Other (1340.6 \pm 721.6 days) than for Caucasians (770.1 \pm 569.4 days; $\mathrm{P}=0.0003)$. Posttransplant BMI was higher in Caucasians compared with other groups. Mean values for 1-week BUN and posttransplant creatinine at discharge, 1 week and 3 years were significantly higher in African-Americans compared with Caucasians.

\section{Relationship Between Patient Race and Patient or Graft Survival}

Patient survival probability after transplantation was similar for patients across the four groups (Figure 3A). At 1, 3 and 5 years posttransplant, patient survival rates by race also were similar (Table $3 \mathrm{~A}$ ). The mean patient survival time was slightly higher in Caucasians (4.23 years) compared with African-Americans (3.43 years),
Hispanics (3.75 years) and Other (2.65 years), but this was not statistically significant (Table 3C).

Graft survival probability was higher in Caucasians compared with African-Americans and Other (Figure $3 \mathrm{~B}, \quad \mathrm{P}<0.05$ for Caucasian vs. African-American and Caucasian vs. Other). Although graft survival was similar between the two races at 1 and 3 years posttransplant (Table 3B), there was a significant decrease in graft survival rate in African-Americans at 5 years compared with Caucasians $(\mathrm{P}=0.02)$. Specifically, $85.3 \%$ of Caucasians had graft survival at 5 years compared with $66.7 \%$ of African-Americans, $97.6 \%$ of Hispanics and $66.7 \%$ of Other at the same time point. The mean graft survival time for Caucasians (3.6 years), Other (5.4 years) and Hispanics (3.4 years) also was higher compared with African-Americans (2.0 years) (Table $3 \mathrm{C}$ ), although this difference did not reach statistical significance $(\mathrm{P}=0.26)$. 
Table 3. 1-, 3- and 5-year patient (A) and graft (B) survival rates among all races (with respective $P$-values comparing proportions of patients who died or suffered graft loss per year across the races separately). Comparative statistics for those who died or suffered graft loss (in terms of years) were computed (C) and showed no statistical significance among races.

\begin{tabular}{|c|c|c|c|c|c|c|c|c|c|c|c|c|c|}
\hline \multirow{2}{*}{$\begin{array}{l}\text { A } \\
\text { Follow-up }\end{array}$} & \multicolumn{3}{|c|}{ CAU $(\mathrm{N}=186)$} & \multicolumn{3}{|c|}{$\mathrm{AA}(\mathrm{N}=59)$} & \multicolumn{3}{|c|}{ HIS $(\mathrm{N}=44)$} & \multicolumn{3}{|c|}{ Other $(\mathrm{N}=18)$} & \multirow[b]{2}{*}{$P^{*}$} \\
\hline & Died & $\begin{array}{l}\text { Pts at } \\
\text { risk }\end{array}$ & Survival & Died & $\begin{array}{l}\text { Pts at } \\
\text { risk }\end{array}$ & Survival & Died & $\begin{array}{c}\text { Pts at } \\
\text { risk }\end{array}$ & Survival & Died & $\begin{array}{c}\text { Pts at } \\
\text { risk }\end{array}$ & Survival & \\
\hline 1 year & 6 & 178 & $96.6 \%$ & 1 & 58 & $98.3 \%$ & 0 & 44 & $100 \%$ & 1 & 17 & $94.1 \%$ & 0.5111 \\
\hline 3 year & 10 & 148 & $93.2 \%$ & 3 & 51 & $94.1 \%$ & 3 & 34 & $91.2 \%$ & 2 & 16 & $87.5 \%$ & 0.8063 \\
\hline 5 year & 17 & 73 & $76.7 \%$ & 7 & 30 & $76.7 \%$ & 4 & 28 & $85.7 \%$ & 2 & 16 & $87.5 \%$ & 0.6165 \\
\hline \multirow{2}{*}{$\begin{array}{l}\text { B } \\
\text { Follow-up }\end{array}$} & \multicolumn{3}{|c|}{ CAU $(\mathrm{N}=186)$} & \multicolumn{3}{|c|}{$\mathrm{AA}(\mathrm{N}=59)$} & \multicolumn{3}{|c|}{ HIS (N=44) } & \multicolumn{3}{|c|}{ Other $(\mathrm{N}=18)$} & \\
\hline & $\begin{array}{l}\text { Graft } \\
\text { lost }\end{array}$ & $\begin{array}{c}\text { Pts at } \\
\text { risk }\end{array}$ & $\begin{array}{c}\text { Graft } \\
\text { survival }\end{array}$ & $\begin{array}{l}\text { Graft } \\
\text { lost }\end{array}$ & $\begin{array}{l}\text { Pts at } \\
\text { risk }\end{array}$ & $\begin{array}{c}\text { Graft } \\
\text { survival }\end{array}$ & $\begin{array}{l}\text { Graft } \\
\text { lost }\end{array}$ & $\begin{array}{c}\text { Pts at } \\
\text { risk }\end{array}$ & $\begin{array}{c}\text { Graft } \\
\text { survival }\end{array}$ & $\begin{array}{l}\text { Graft } \\
\text { lost }\end{array}$ & $\begin{array}{c}\text { Pts at } \\
\text { risk }\end{array}$ & $\begin{array}{c}\text { Graft } \\
\text { survival }\end{array}$ & $P^{*}$ \\
\hline 1 year & 4 & 177 & $97.7 \%$ & 2 & 52 & $96.2 \%$ & 1 & 42 & $97.6 \%$ & 1 & 17 & $94.1 \%$ & 0.7948 \\
\hline 3 year & 7 & 122 & $94.3 \%$ & 6 & 42 & $85.7 \%$ & 1 & 42 & $97.6 \%$ & 1 & 17 & $94.1 \%$ & 0.1512 \\
\hline 5 year & 11 & 75 & $85.3 \%$ & 9 & 27 & $66.7 \%$ & 1 & 42 & $97.6 \%$ & 2 & 6 & $66.7 \%$ & $<0.005$ \\
\hline \multirow[t]{2}{*}{ C } & & \multicolumn{5}{|c|}{ Time to death, years } & & \multicolumn{5}{|c|}{ Time to graft loss, years } & \\
\hline & & $\begin{array}{c}\text { CAU } \\
(\mathrm{N}=27)\end{array}$ & $\begin{array}{c}\text { AA } \\
(\mathrm{N}=9)\end{array}$ & $\begin{array}{c}\mathrm{HI} \\
(\mathrm{N}=\end{array}$ & & $\begin{array}{l}\text { Other } \\
(\mathrm{N}=3)\end{array}$ & $P$ & $\begin{array}{r}\text { CAU } \\
(\mathrm{N}=15\end{array}$ & $\begin{array}{c}\text { AA } \\
(\mathrm{N}=9)\end{array}$ & & $\begin{array}{l}\text { HIS } \\
(\mathrm{N}=3)\end{array}$ & $\begin{array}{l}\text { Other } \\
(\mathrm{N}=4)\end{array}$ & $\boldsymbol{P}$ \\
\hline Mean & & 4.23 & 3.43 & 3.7 & & 2.65 & 0.754 & 3.6 & 2 & & 3.4 & 5.4 & 0.267 \\
\hline SD & & 3.1 & 2.1 & 1. & & 3.5 & & 3.1 & 1.3 & & 2.9 & 4.1 & \\
\hline Median & & 3.6 & 3.6 & 3. & & 1.2 & & 3.5 & 1.89 & & 5.04 & 6 & \\
\hline Minimum & & 0.11 & 0.24 & 1.1 & & 0.21 & & 0.03 & 0.31 & & 0.02 & 0.21 & \\
\hline Maximum & & 11.12 & 6.79 & 6.1 & & 6.6 & & 9.19 & 3.87 & & 5.04 & 9.21 & \\
\hline
\end{tabular}

${ }^{*} P<0.05$ denoted significance.

AA, African-American; CAU, Caucasian; HIS, Hispanic; Pts, patients; SD, standard deviation.

Figure 4. Hazard ratios for variables found to be significant for all races in terms of patient and graft survival in a stepwise regression. BUN, blood urea nitrogen; $\mathrm{Cl}$, confidence interval; CVA, cardiovascular accident; HF, heart failure; HepC, hepatitis C; MI, myocardial infarction.

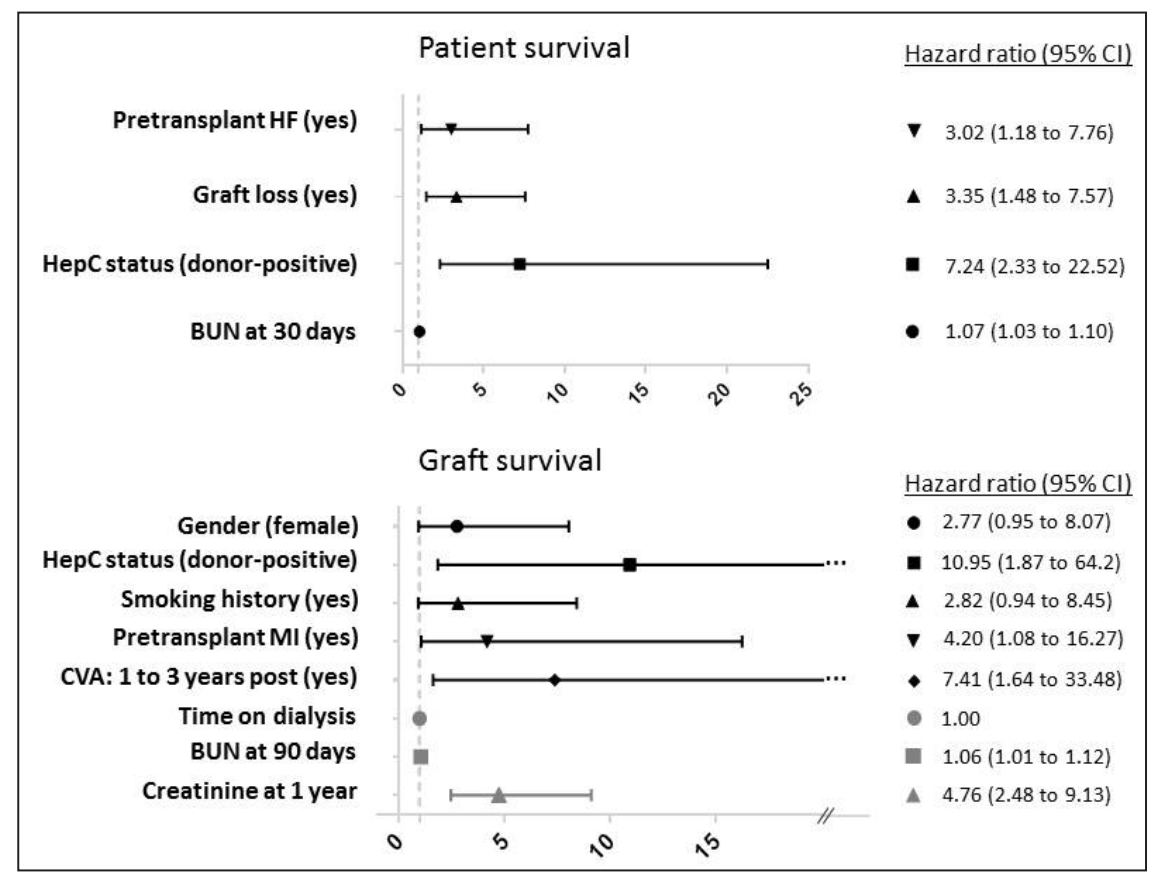




\section{Factors Impacting Patient and Graft Survival}

All factors that were associated with patient death by univariate analysis are presented in Table 4, which shows that patient survival was multifactorial and depended on a variety of patient factors such as age, type of transplant (cadaveric vs. living), serum creatinine and BUN levels, and comorbidities such as diabetes, coronary artery disease, myocardial infarction and heart failure. Cox regression modeling for multivariate analysis indicated that preprocedure heart failure, graft loss, HepC-positive donor status and high 30-day BUN were significant risk factors for patient survival. The hazard ratio (HR) for each one of these factors is shown in Figure 4. Most notably, heart failure conferred a threefold risk, graft loss a 3.35-fold risk and HepC-positive donor a sevenfold risk for early patient death.

For the secondary outcome of graft survival, univariate analysis showed that graft survival also was multifactorial (Table 4). Multivariate analysis indicated that female gender (HR: 2.77), HepCpositive donor status (HR: 10.95), smoking history (HR: 2.82), preprocedure myocardial infarction (HR: 4.2), cerebrovascular accident at $1-3$ years (HR: 7.41) and 1-year elevated serum creatinine (HR: 4.76) were independent risk factors for graft loss.

\section{DISCUSSION}

In examining the factors affecting patient and graft survival following kidney transplantation across Caucasians, African-Americans, Hispanics and other races, we found that patient survival rates were similar at 1,3 and 5 years posttransplantation. However, African-Americans and Other had significantly lower graft survival at 5 years posttransplant compared with Caucasians and Hispanics. On average, the time to patient death was shorter in African-Americans and Other compared with Caucasians and the time to graft loss also was shorter in African-Americans compared with the rest of the groups, though these differences were not found to be statistically significant. In the end both patient death and graft loss were multifactorial, and no single factor stood out as being independently associated with either outcome. Female gender, HepCpositive donor, smoking history, myocardial infarction and cerebrovascular accident all were associated with graft loss.
Table 4. Significant variables for patient and graft survival used in multivariate analysis $(P<0.05)$

\begin{tabular}{|c|c|}
\hline (A) Patient Survival & (B) Graft Survival \\
\hline $\begin{array}{l}\text { Demographics } \\
\text { Gender } \\
\text { Age (years) }\end{array}$ & $\begin{array}{l}\text { Demographics } \\
\text { Gender } \\
\text { Smoking history }\end{array}$ \\
\hline $\begin{array}{l}\text { Clinical data } \\
\text { 30-day BUN } \\
\text { 3-month BUN } \\
\text { Donor hepatitis C } \\
\text { 1-year BUN } \\
\text { 3-year BUN* } \\
\text { Length of stay } \\
\text { Pretransplant creatinine } \\
\text { 3-year creatinine* } \\
\text { Transplant type } \\
\text { Pretransplant GFR* } \\
\text { 3-month GFR* } \\
\text { Graft loss } \\
\text { 1-year GFR* } \\
\text { 3-year GFR* } \\
\text { Medication adherence } \\
\text { 1-year tacrolimus* }\end{array}$ & $\begin{array}{l}\text { Clinical data } \\
\text { 1-week creatinine } \\
\text { 30-day creatinine } \\
\text { Donor hepatitis C } \\
\text { 3-month creatinine } \\
\text { 1-year creatinine } \\
\text { Length of stay } \\
\text { 3-year creatinine* } \\
\text { 1-week BUN } \\
\text { Time on dialysis } \\
\text { 30-day BUN } \\
\text { 3-month BUN } \\
\text { Medication adherence } \\
\text { 1-year BUN } \\
\text { 3-year BUN* } \\
\text { 1-month tacrolimus* } \\
\text { 1-week GFR* }\end{array}$ \\
\hline $\begin{array}{l}\text { Comorbidities } \\
\text { Pretransplant DM } \\
\text { Pretransplant HF } \\
\text { Pretransplant HLD } \\
\text { Pretransplant MI } \\
\text { Pretransplant CAD } \\
\text { HF: } 0-1 \text { years post } \\
\text { CAD: } 0-1 \text { years post } \\
\text { CVA: } 0-1 \text { years post }\end{array}$ & $\begin{array}{l}\text { 30-day GFR* } \\
\text { 1-year tacrolimus* } \\
\text { 3-month GFR* } \\
\text { 1-year GFR* } \\
\text { 3-year tacrolimus* } \\
\text { 3-year GFR* } \\
\text { Comorbidities } \\
\text { Pretransplant CVA } \\
\text { Pretransplant CAD } \\
\text { Pretransplant MI } \\
\text { Pretransplant HF } \\
\text { Pretransplant DM } \\
\text { HF: 0-1 years post } \\
\text { CVA: } 1-3 \text { years post }\end{array}$ \\
\hline
\end{tabular}

${ }^{*}$ Denotes variables with less than $80 \%$ complete data (not included in multivariate analysis). All variables were examined for univariate analysis; however, only statistically significant variables are presented.

$B U N$, blood urea nitrogen; $C A D$, coronary artery disease; CVA, cerebrovascular accident; DM, diabetes mellitus; GFR, glomerular filtration rate; HF, heart failure; MI, myocardial infarction.

Overall patient outcomes in our study were comparable to national averages for patient and graft survival, and our 5-year graft survival rate was $15 \%$ higher than data from the National Institutes of Health published in 2012. ${ }^{23}$ However, despite the overall success of kidney transplants, African-Americans 
and patients in the Other racial/ethnic group had poorer graft outcomes 5 years after transplantation compared with Caucasian patients. Even though overall access to health care and availability of immunosuppressants have improved nationally, racial disparity in graft survival still exists as shown in past studies. ${ }^{24,25}$ Racial differences in genotypes or gene expressions like angiotensin receptors, transforming growth factor- $\beta$, etc. ${ }^{26,27}$ and socioeconomic factors such as decreased access to health care and lower income status adversely affect transplant outcomes in African-Americans. ${ }^{28}$

In this regard, our study showed that AfricanAmericans had a significantly longer time on dialysis compared with Caucasians, which could be related to limited access to transplants in African-Americans due to greater delays in referral to transplant centers and longer waiting times for transplants. Interestingly, our data also showed that while only $6.6 \%$ of Caucasians had more than 5 years of dialysis, all other groups were higher, with AfricanAmericans at $17 \%$, Hispanics at $9.5 \%$ and Other at $31.3 \%$ of their respective populations. While the high proportion of Other with high dialysis times could be explained by immunologic factors and availability of histocompatibility matches, the high dialysis times in African-Americans and Hispanics are more likely related to poor access to transplants. Recently a community-based participatory research trial called RADIANT was launched with the purpose of reducing disparities in access to kidney transplantation in the states of Georgia, North Carolina and South Carolina. ${ }^{29}$ The results of this trial will inform future educational interventions and studies to monitor access to transplantation in our center.

Although we did not study immunologic factors contributing to worse graft survival in AfricanAmericans, we did find nonimmunologic differences such as a greater proportion of AfricanAmericans and Hispanics with higher creatinine and BUN levels pretransplant, at discharge and at 1 week posttransplant. The poor pretransplant kidney functional status seen in African-Americans also was likely related to the higher incidence of pretransplant diabetes compared with Caucasians. Although our analysis did not indicate a clear relationship between these factors and patient/graft survival, it is likely that the presence of higher comorbidities in AfricanAmericans results in worse outcomes.

Overall, patient and graft survival was dependent on several factors and no one variable could be singled out as contributing to poorer outcomes in transplant recipients across the different races. Female gender was associated with graft loss, which could be related to increased female-male donations compared with male-female donations. ${ }^{30}$ Designing studies to take into account the gender of donors and recipients might be an important consideration in the future. Our study showed that certain comorbidities, such as presence of preprocedure heart failure, were associated with patient death (HR: 3.02), and preprocedure myocardial infarction and cerebrovascular accident at 1-3 years postprocedure both contributed to poor graft outcomes (HR: 4.2 and 7.41, respectively). While these comorbidities were distributed more or less equally across racial/ethnic groups, all are modifiable and can be linked to lifestyle and behavior. For example, smoking history was associated with graft loss (HR 2.82), and this behavior is modifiable.

\section{Study Limitations}

The overall sample size of our population cohort did not allow for comparison when subdivided into individual races. Although African-Americans were well-represented proportionately (19\%), the total number was small (59 patients). Also, we were unable to obtain all necessary follow-up data, as not all patients stayed within our institution's health system after transplantation or were lost to followup. Finally, there were a number of variables for which more than $20 \%$ of electronic health record information was missing, limiting the analysis and interpretation of results.

\section{CONCLUSIONS}

Our study showed that disparities exist in graft outcomes across different racial/ethnic groups within a single center, despite overall kidney transplant success rates on par with national averages. The causes of graft loss are multifactorial. Prospective interventional studies with strategies to improve graft function and survival rates for kidney transplantation in AfricanAmericans and other minorities are warranted. 


\section{Patient-Friendly Recap}

- The best treatment for patients with serious renal disease is kidney transplantation.

- Success rates of these transplants vary among different races and ethnicities.

- The authors analyzed a series of patient and disease factors to identify possible contributors to population disparities.

- They found that African-American patients had more time on dialysis, higher pretransplant creatinine levels and higher BMI compared to Caucasian patients, all of which may contribute to their higher rate of graft failure.

\section{Conflicts of Interest}

None.

\section{References}

1. Wolfe RA, Ashby VB, Milford EL, et al. Comparison of mortality in all patients on dialysis, patients on dialysis awaiting transplantation, and recipients of a first cadaveric transplant. N Engl J Med. 1999;341:1725-30. CrossRef

2. Guedes AM, Malheiro J, Fonseca I, et al. Over ten-year kidney graft survival determinants. Int J Nephrol. 2012;2012:302974. CrossRef

3. Palmer Alves T, Lewis J. Racial differences in chronic kidney disease (CKD) and end-stage renal disease (ESRD) in the United States: a social and economic dilemma. Clin Nephrol. 2010;74 Suppl 1:S72-7.

4. Peters TG. Good news regarding race and kidney transplant access in America. Am J Transplant. 2012;12:810-1. CrossRef

5. Malek SK, Keys BJ, Kumar S, Milford E, Tullius SG. Racial and ethnic disparities in kidney transplantation. Transpl Int. 2011;24:419-24. CrossRef

6. Ayanian JZ, Cleary PD, Weissman JS, Epstein AM. The effect of patients' preferences on racial differences in access to renal transplantation. N Engl J Med. 1999;341:1661-9. CrossRef

7. Eggers PW. Racial differences in access to kidney transplantation. Health Care Financ Rev. 1995;17:89-103.

8. 2009 Annual Report of the U.S. Organ Procurement and Transplantation Network and the Scientific Registry of Transplant Recipients: Transplant Data 1998-2008. Rockville, MD: U.S. Department of Health and Human Services, Health Resources and Services Administration, Healthcare Systems Bureau, Division of Transplantation, 2009.

9. Young CJ, Gaston RS. African Americans and renal transplantation: disproportionate need, limited access, and impaired outcomes. Am J Med Sci. 2002;323:94-9. CrossRef

10. Organ Procurement and Transplantation Network and Scientific Registry of Transplant Recipients 2010 data report. Am J Transplant. 2012;12 Suppl 1:1-156. CrossRef

11. Cosio FG, Dillon JJ, Falkenhain ME, et al. Racial differences in renal allograft survival: the role of systemic hypertension. Kidney Int. 1995;47:1136-41. CrossRef
12. Yeates K, Wiebe N, Gill J, et al. Similar outcomes among black and white renal allograft recipients. J Am Soc Nephrol. 2009;20:172-9. CrossRef

13. Isaacs RB, Nock SL, Spencer CE, et al. Racial disparities in renal transplant outcomes. Am J Kidney Dis. 1999;34:706-12. CrossRef

14. Gordon EJ, Caicedo JC. Ethnic advantages in kidney transplant outcomes: the Hispanic Paradox at work? Nephrol Dial Transplant. 2009;24:1103-9. CrossRef

15. Ladin K, Hanto DW. Understanding disparities in transplantation: do social networks provide the missing clue? Am J Transplant. 2010;10:472-6. CrossRef

16. Ladin K, Rodrigue JR, Hanto DW. Framing disparities along the continuum of care from chronic kidney disease to transplantation: barriers and interventions. Am J Transplant. 2009;9:669-74. CrossRef

17. Feyssa E, Jones-Burton C, Ellison G, Philosophe B, Howell C. Racial/ethnic disparity in kidney transplantation outcomes: influence of donor and recipient characteristics. J Natl Med Assoc. 2009;101:111-5.

18. Chakkera HA, O'Hare AM, Johansen KL, et al. Influence of race on kidney transplant outcomes within and outside the Department of Veterans Affairs. J Am Soc Nephrol. 2005;16:269-77. CrossRef

19. Young CJ, Gaston RS. Understanding the influence of ethnicity on renal allograft survival. Am J Transplant. 2005;5:2603-4. CrossRef

20. Goldfarb-Rumyantzev AS, Koford JK, Baird BC, et al. Role of socioeconomic status in kidney transplant outcome. Clin J Am Soc Nephrol. 2006;1:313-22. CrossRef

21. Fan PY, Ashby VB, Fuller DS, et al. Access and outcomes among minority transplant patients, 1999-2008, with a focus on determinants of kidney graft survival. Am J Transplant. 2010;10:1090-107. CrossRef

22. Gordon EJ, Ladner DP, Caicedo JC, Franklin J. Disparities in kidney transplant outcomes: a review. Semin Nephrol. 2010;30:81-9. CrossRef

23. National Kidney and Urologic Diseases Information Clearinghouse. Kidney Disease Statistics for the United States (NIH Publication No. 12-3895, June 2012). Bethesda, MD: National Institutes of Health, 2012, p. 10.

24. Eckhoff DE, Young CJ, Gaston RS, et al. Racial disparities in renal allograft survival: a public health issue? J Am Coll Surg. 2007;204:894-902; discussion 902-3. CrossRef

25. Young CJ, Gaston RS. Renal transplantation in black Americans. N Engl J Med. 2000;343:1545-52. CrossRef

26. August P, Sharma V, Ding R, Schwartz JE, Suthanthiran M. Transforming growth factor beta and excess burden of renal disease. Trans Am Clin Climatol Assoc. 2009;120:61-72.

27. Klahr S, Morrissey J. Angiotensin II and gene expression in the kidney. Am J Kidney Dis. 1998;31:171-6. CrossRef

28. Nee R, Jindal RM, Little D, et al. Racial differences and income disparities are associated with poor outcomes in kidney transplant recipients with lupus nephritis. Transplantation. 2013;95:1471-8. CrossRef

29. Patzer RE, Gander J, Sauls L, et al. The RaDIANT community study protocol: community-based participatory research for reducing disparities in access to kidney transplantation. $B M C$ Nephrol. 2014;15:171. CrossRef

30. Tan JC, Wadia PP, Coram M, et al. H-Y antibody development associates with acute rejection in female patients with male kidney transplants. Transplantation. 2008;86:75-81. CrossRef

(C) 2016 Aurora Health Care, Inc. 